\title{
A Case Report of Spontaneous Pneumoperitoneum
}

\author{
${ }^{1}$ Department of General Surgery, Lithuania \\ ${ }^{2}$ Department of Surgery, Lithuania \\ ${ }^{3}$ Department of Gastroenterology, Lithuania \\ *Corresponding author: Saulius Bradulskis, Department of General Surgery, Lithuania
}

Saulius Bradulskis ${ }^{1 *}$, Lina Pankratjevaite ${ }^{2}$, Kestutis Adamonis $^{3}$, Donatas Venskutonis ${ }^{1}$ and Egle Virbickaite ${ }^{2}$

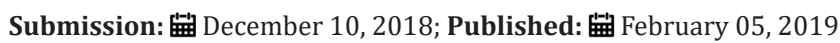

\begin{abstract}
Introduction: Pneumoperitoneum can have many causes (thoracic, abdominal, gynaecological, iatrogenic) or sometimes can be idiopathic. In most of the cases it is associated with perforation of hollow viscera and usually requires an urgent surgical intervention. In rare cases, pneumoperitoneum might be non-surgical (there is no visceral perforation and no surgical treatment is required).

Case report: We present a case report about spontaneous pneumoperitoneum after fibro esophagogastroduodenoscopy (FEGDS). An 88-yearold woman was hospitalized to the Intensive Care Unit because of hypovolemic shock due to severe gastrointestinal bleeding. FEGDS was performed and the bleeding was stopped. Next day the patient was suspected to have pneumonia and chest x-ray was done. The X-ray has revealed free air in peritoneal cavity. Although, there were no signs of peritonitis, an urgent laparotomy was done. However, during the surgery no cause of pneumoperitoneum was found.
\end{abstract}

Conclusion: It is very important to know a comprehensive patient's illness history, to do thorough physical examination, appropriate laboratory tests and radiologic examination to identify patients with non-surgical pneumoperitoneum and avoid unnecessary operation.

Keywords: Pneumoperitoneum; Non-surgicalpneumoperitoneum;Idiopathicspontaneouspneumoperitoneum;Fibroesophagogastroduodenoscopy; Surgery

Core Tips

Pneumoperitoneum might be surgical and non-surgical. It is very important to comprehensively evaluate the clinical situation to avoid unnecessary operation. We report a case about spontaneous pneumoperitoneum after FEGDS. During the surgery no cause of pneumoperitoneum was found. Appealing to our experience and analysis of the literature, we claim that free air in peritoneal cavity after endoscopic procedure is a very rare case. We consider that in ambiguous clinical situations laparoscopic surgery could be a standard procedure to diagnose the aetiology of pneumoperitoneum, and to perform treatment manipulations.

\section{Introduction}

The term pneumoperitoneum describes the presence of free air within the peritoneal cavity. Pneumoperitoneum can be surgical and non-surgical $[1,2]$. In most of the cases (about 90 percent) it is associated with the perforation of the hollow viscera, when the only treatment is an urgent surgery $[3,4]$. In the other 10 percent of the cases pneumoperitoneum is not associated with the hollow viscera perforation and the causes of it are various: thoracic, abdominal, gynaecological, iatrogenic or very rarely it can be idiopathic [3]. Pneumoperitoneum is diagnosed with abdominal x-ray (free air in the abdominal cavity is detected) [5]. However, pneumoperitoneum might be seen just in 85 percent of the abdominal x-ray when the perforation of the hollow viscera is present [5]. In other cases, a computed tomography (CT) scan may be done - it can reveal free air in peritoneal cavity in up to $95 \%$ of the cases with a ruptured hollow viscerus [5].
If free air in the cavity is detected, doctor must carefully evaluate patient's history, clinical situation, laboratory test results. These steps are very important because doctor must make a decision about patient's treatment: does the illness require surgery or conservative treatment is enough. The perforation of the hollow viscera requires an urgent surgical intervention [1]. But the presence of pneumoperitoneum with no evidence of the perforation and without the signs of peritonitis does not require laparoscopy or laparotomy [2]. However, there are some clinical situations which can cause diagnostic difficulties. For example, it was not found any cause of free air in the abdomen after the diagnostic endoscopic procedure. Firstly, that type of clinical situation is rare, secondly, to eliminate complication after endoscopy is very difficult. We present a case report about pneumoperitoneum after fibro esophagogastroduodenoscopy. An urgent laparotomy was done the next day, but no cause of pneumoperitoneum was found. 


\section{Case Report}

An 88-year-old woman was hospitalized urgently to the Intensive Care Unit because of hypovolemic shock due to severe gastrointestinal bleeding. Her past medical history was significant for ischemic heart disease, unstable chest angina, atrial fibrillation and Orfarin use. During the initial physical examination her blood pressure was $70 / 40 \mathrm{mmHg}$, heart rate 100 per min. Her skin and visible mucous were pale, there was no tenderness of abdomen. Digital examination per rectum revealed melena. Blood laboratory findings were: haemoglobin - 74g/l, red blood cells (RBC) - 2.77x1012/l, potassium - 4.3mmol/l, prothrombin time 6 percent, white blood cells (WBC) - 7.4x109/l, C reactive protein (CRP) $-2.20 \mathrm{mg} / \mathrm{l}$. Hypovolemia was corrected by the transfusion of three units of red blood cells and crystalloid solution. To correct coagulation system disorder were injected 4 doses of Octaplex 500IU. There were repeated blood laboratory tests: haemoglobin 88, RBC - 2.89x1012/l, prothrombin time - 54 percent, International Normalised Ratio (INR) - 1.34. However, haematin with addition of fresh blood was leaking through the probe which was introduced to the stomach. Then FEGDS without sedation was performed. It revealed multiple haemorrhagic erosions, most of the area of the stomach body. There was slow bleeding from the erosive mucous of the stomach minor curve below the corner.

Haematin and not much fresh blood were seen in the stomach. Pylorus and duodenum were without any changes. $10 \mathrm{ml}$ of sol. Adrenaline ( 0.1 percent) was injected to the stomach antrum beside the minor curve to stop the bleeding. The bleeding has stopped. After that the patient was treated conservatively. Her condition was stable; she had no pain of abdomen. Next day the patient was suspected to have pneumonia due to the occurring failure in the respiratory system (her saturation decreased to 88 percent without oxygen and the respiratory rate has increased to 22 times per min). Chest $\mathrm{x}$-ray was done. It denied pneumonia diagnosis but revealed free air in peritoneal cavity (Figure 1).

The chest $\mathrm{x}$ ray was repeated but the findings were the same. During the physical examination patientabdomen was a little distent, painful around the umbilicus, but without any signs of peritonitis. Blood test has showed: WBC - 37.1 x109/l, CRP - 18.0mg/l. There was no way to eliminate hollow viscera perforation. The urgent laparotomy was done. A lot of air in peritoneal cavity was found during the operation (the gas went outside from the peritoneal cavity with pressure after the incision of abdominal wall was done). Small haematoma and infiltration were seen in the minor curve of the stomach (probably it was the zone of injected Adrenaline during FEGDS). No hollow viscera perforation was found. Stomach and duodenum were mobilised and $2000 \mathrm{ml}$ of physiologic liquid were poured to the abdominal cavity. The air was insufflated through the nasogastric probe. No air bubbles were seen. Peritoneal cavity was drained and incision of abdomen was sutured. The patient was treated in ICU next few days. The patient hemodynamic was stable, her respiratory system function got better. Next day after the surgery patient's WBC has decreased to $16,9 \times 109 / 1$, later to 9,3 x109/l. Haemoglobin has rised to $104 \mathrm{~g} / \mathrm{l}$, later to $132 \mathrm{~g} / \mathrm{l}$. Twelve days after the surgery patient was discharged from the hospital (Figure 1).

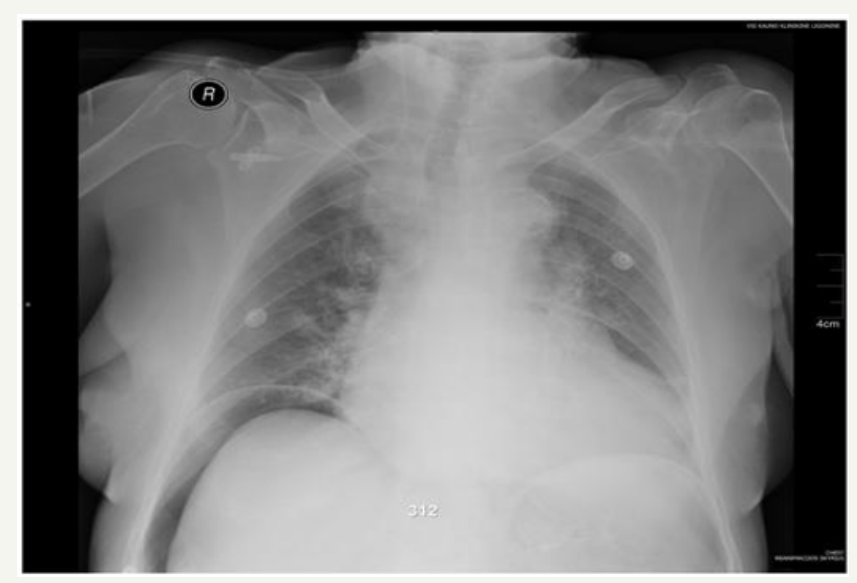

Figure 1: Chest x-ray showing pneumoperitoneum.

\section{Discussion}

All reasons for pneumoperitoneum fall in two groups: surgical and non-surgical [1,2]. Surgical reasons are associated with perforation of the hollow viscera: it is about 90 percent of the cases when free air is found in the abdominal cavity [3,4]. Spontaneous pneumoperitoneum can have thoracic (i.e. intermittent positivepressure ventilation, barotrauma, bronchopneumonic fistula, pneumothorax) [4-6], abdominal (i.e. ruptured intraperitoneal abscess [7], pneumatosis cystoides intestinalis [8]), gynaecological causes (i.e. sexual activity [3,9], gynaecological examination [6]) or can be iatrogenic - air originating after endoscopic procedures [2]. According to the literature, pneumoperitoneum can be idiopathic too: the risk factors of this condition are not clear and even after the laparotomy the reasons for it are not found [3]. It is very important to diagnose true pneumoperitoneum cause and to give appropriate treatment: to understand when it requires urgent surgical intervention, or when conservative treatment is enough. When pneumoperitoneum is associated with perforation, the treatment is conventional - urgent surgery for elimination of the perforation $[3,6,10]$. Surgery can be traditional laparotomy or minimally invasive. Some authors suggest performing laparoscopic exploration instead of laparotomy: both can determine and treat the cause, but laparoscopic procedure has minimally invasive surgery advantages [1]. Of course, non-surgical pneumoperitoneum requires conservative treatment: surgical exploration in this case is not needed and can cause various complications [5].

When free air in abdomen is present, but there are no any signs of peritonitis, and there is no evidence or perforation (all causes which can require doing an exploratory laparotomy are denied), some authors suggest to manage the patient conservatively: to watch their condition and to do surgery just when the inflammation markers are increasing $[2,10,11]$. Spontaneous pneumoperitoneum is associated in most cases with other severe illness and unnecessary laparotomy can worsen the patient condition [11]. There are successful conservative treatment examples in literature 
$[11,12]$. Some authors say that idiopathic pneumoperitoneum even presented with signs and symptoms of peritonitis can be successfully managed by conservative treatment [12].

Some sources of the literature recommend to deny surgical intervention when peritonitis signs and symptoms are not present $[2,10]$. But there are some situations when evaluating/evaluation of these symptoms could be very difficult. If the patient is suppressed by medications and cannot breathe on his own (intubation and mechanical ventilation is used), when ascites or colon perforation are present, the doctor can do a mistake evaluating the pain of patient abdomen or muscles tense. Strict vigilance must be performed in patients with pneumoperitoneum, when clinical examination is improper and peritonitis signs may be occult [6]. It is very important to know the patient 's history thoroughly, to do comprehensive physical examination, appropriate laboratory tests and radiologic examination to identify patients with nonsurgical pneumoperitoneum. Moreover, doctor must know that non-surgical pneumoperitoneum can be asymptomatic or to have abdomen pain [5].

Plain or lateral decubitus abdomen or chest $\mathrm{x}$ ray can help diagnose pneumoperitoneum: it shows free air within the peritoneal cavity under the diaphragm [2], as it was in our case. Non-surgical pneumoperitoneum in most cases is founded accidentally: when for some reasons chest $\mathrm{x}$ ray is done (i.e. to diagnose pneumonia) [5]. It is important to mention that pneumoperitoneum is visible on abdominal x-ray just in 85-89 percent of the cases with a rupture of a hollow organ $[5,13]$. When perforation is suspected but pneumoperitoneum is not visible on abdominal $\mathrm{x}$-ray, a computer tomography may be done: it can reveal free air in peritoneal cavity in up to 95 percent of cases with a ruptured hollow viscerus [5] Furthermore, strict attention must be given evaluating $\mathrm{x}$-ray for pseudopneumoperitoneum: particular pathologies (such as overdistension of hollow viscera, undulant configuration of the diaphragm, interposition of the colon hepatic flexure between the right lobe of the liver and the diaphragm, etc.) can simulate pneumoperitoneum [10].

Endoscopic procedures are associated with pneumoperitoneum too [2,3]. Perforation of gastrointestinal tract is well known endoscopic examination complication [3]. Free air can occur in peritoneal cavity without macroscopic perforation of hollow organ after endoscopic procedures such as gastroscopy or colonoscopy [10]. In that case pneumoperitoneum probably occurs because of high intraluminal pressure which causes microscopic perforations [10]. Air escapes through the microperforations, but intestinal content stays in the lumen [5]. Pneumoperitoneum may be detected in up to 0.1 percent after the endoscopic procedures [6]. Spontaneous pneumoperitoneum after endoscopic procedure is very rare occasion, when endoscopic mucosal resection, endoscopic submucosal dissection is performed. There are few articles about pneumoperitoneum after FEGDS when organ perforation was not found during surgery $[11,14,15]$. It should be noted that all these endoscopic procedures were performed with semi-rigit endoscope.
About pneumoperitoneum after "technically difficult" gastroscopy we have found just few articles [16,17]. In our opinion, the origin of pneumoperitoneum after endoscopic procedure is very specific and complicated case [18].

Endoscopy is not very safe procedure. To guarantee, that there is no perforation after the procedure is difficult. Peptic ulcer is very often found during endoscopy and itself can be the reason of perforation. Additional diagnostic manipulations such as injection of air to gastrointestinal tract and repeated x-ray and CT are not useful. If air amount in abdomen does not increase it does not mean there is no perforation. Normally we can compare air amount in the abdomen visually by comparing together two abdominal plain x-ray photos. Repeated FEGDS can help to evaluate changes just in the stomach and duodenum. Besides it does not deny other pathology like perforation of diverticula which are more distal. Moreover, if there is an organ perforation, the air amount in abdomen increases catastrophically during endoscopic and it can worsen patient's condition. Above mentioned diagnostic difficulties shows our case. Patient's abdomen was painful but without peritonitis signs, there were increased markers of inflammation. The amount of free air founded during surgery was substantial That is why it is difficult to think about spontaneous pneumoperitoneum. But to estimate that air originated to peritoneal cavity during Adrenalin injection in to the bleeding place is extremely challenging. Our performed surgery was additional trauma to the patient. If pneumoperitoneum originates after endoscopic procedure, we suggest performing a laparoscopy. It has minimally invasive surgery advantages and can help to evaluate changes in abdominal cavity, to diagnose the aetiology of pneumoperitoneum, and to perform treatment manipulations. We think it could be a standard.

\section{Conclusion}

Non-surgical pneumoperitoneum is a rare condition. It is very important to know a thorough patient's history, to do comprehensive physical examination, appropriate laboratory tests and radiologic examination to identify patients with nonsurgical pneumoperitoneum and avoid unnecessary operation.

Appealing to our experience, after analysis of the existing literature, we say that free air in peritoneal cavity after endoscopic procedure is a very rare case. The standard letting to evaluate changes in abdomen could be a laparoscopic surgery. Treatment tactic during laparoscopy depends on findings. Conservative treatment, just with intravenous nutrition for few days could have a major role. In elderly patients, the presence of peumoperitoneum after upper endoscopy could be treated conservatively for few days with a "watch and see" attitude.

\section{References}

1. Pitiakoudis M, Zezos P, Oikonomou A, KirmanidisM, Kouklakis G, et al. (2011) Spontaneous idiopathic pneumoperitoneum presenting as an acute abdomen: a case report. Journal of Medical Case Reports 5: 86.

2. Karaman A, Demirbilek S, Akın M, Gurunluoglu K, Irsi C (2005) Does pneumoperitoneum always require laparotomy? Report of six cases and review of the literature. Pediatr Surg Int 21(10): 819-824. 
3. Williams NMA, Watkin DFL (1997) Spontaneous pneumoperitoneum and other nonsurgical causes of intraperitoneal free gas. Postgrad Med J 73(863): 531-537.

4. Alassaf M (2014) Recurring spontaneous aseptic pneumoperitoneum presenting secondary to an unrelated chief complaint: A case report. Int J Surg Case Rep 7C: 96-98.

5. Čečka F, Sotona O, Šubrt Z (2014) How to distinguish between surgical and non-surgical pneumoperitoneum? Signa Vitae 9(1): 9-15.

6. Mularski RA, Ciccolo ML, Rappaport WD (1999) Nonsurgical causes of pneumoperitoneum. West J Med 170(1): 41-46.

7. Shiba H, Aoki H, Misawa T, Kobayashi S, Saito R, Yanaga K (2007) Pneumoperitoneum caused by ruptured gas-containing liver abscess. J Hepatobiliary Pancreat Surg 14(2): 210-211.

8. Daly BD, Guthrie JA, Couse NF (1991) Pneumoperitoneum without peritonitis. Postgrad Med J 67(793): 999-1003.

9. Jacobs VR, Mundhenke C, Maass N, Hilpert F, Jonat W (2000) Sexual activity as cause for non-surgical pneumoperitoneum. JSLS 4(4): 297 300.

10. Derveaux K, Penninckx F (2003) Recurrent "spontaneous" Pneumoperitoneum: a Diagnostic and Therapeutic Dilemma. Acta Chir Belg 103(5): 490-492.

11. Ylvisaker RS, Myhre J (1951) Management of pneumoperitoneum after gastroscopy. Gastroenterology 18(3): 463-465.
12. Tani T, Shirai Y, Sasagawa M, Wanifuchi T, Yagi K, et al. (1995) Conservative Management of Idiopathic Pneumoperitoneum Masquerading as Peritonitis: Report of a Case. Surg Today 25(3): 265-267.

13. Bansal J, Jenaw RK, Rao J, Kankaria J, Agrawal NN (2012) Effectiveness of plain radiography in diagnosing hollow viscus perforation: study of 1,723 patients of perforation peritonitis. Emerg Radiol 19(2): 115-119.

14.Schindler R (1945) Passage of air through the gastric wall during gastroscopy, with no wound demonstrable three hours later. Gastroenterology.

15.Samach M, Brandt U, Bernstein LH (1978) Spontaneous pneumoperitoneum with pneumatosis cystoides intestinalis in a patien with mixed connective tissue disease. Am J Gastroenterol 69(4): 494500 .

16. Rathous I, Sváb J (2002) Nonsurgical pneumoperitoneum. Rozhl Chir 81(9): 459-463.

17.López-Boado MA, Rull R, Bataller R, González FX, Bordas JM, et al. (1996) Asymptomatic pneumoperitoneum after fiber gastroscopy. Rev Esp Enferm Dig 88(5): 365-367.

18.Gagnier JJ, Kienle G, Altman DG, Moher D, the CARE Group, et al (2013) The CARE Guidelines: Consensus-based Clinical Case Reporting Guideline Development. BMJ Case Rep.
Creative Commons Attribution 4.0

International License

For possible submissions Click Here
Submit Article

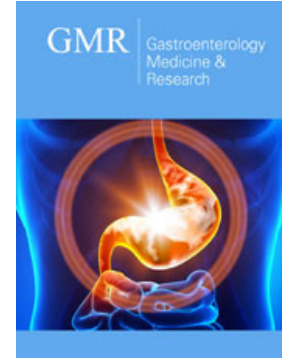

\section{Gastroenterology Medicine \& Research}

\section{Benefits of Publishing with us}

- High-level peer review and editorial services

- Freely accessible online immediately upon publication

- Authors retain the copyright to their work

- Licensing it under a Creative Commons license

- Visibility through different online platforms 